\title{
Vitamin D status in children with migraine: a case-control study
}

\author{
Aliakbar Momen', Kourosh Riahi', Gholamreza Jelodar', Shahram Nasiri', \\ Sahar Majidinezjad ${ }^{1}$, Hamid Nilsaz-Dezfouli², Reza Azizimalamiri ${ }^{1}$ \\ 'Department of Pediatrics, Golestan Medical, Educational, and Research Center, Ahvaz Jundishapur University of Medical \\ Sciences, Ahvaz, Iran \\ 2Department of Industrial Engineering, Islamic Azad University, Mahshahr, Iran
}

\section{ABSTRACT}

Introduction: Vitamin D deficiency has been observed in patients with migraine; however, the results of previous studies are controversial. We aimed to evaluate vitamin D status in a group of children with migraine and to compare them with a group of healthy control.

Material and methods: We conducted a prospective case-control study to assess the vitamin D status in children with migraine comparing them with age- and sex-matched healthy controls. Thirty children with migraine and 30 healthy controls were enrolled in the study. The age range was 6-14 years. The vitamin D status was classified as optimal (> $20 \mathrm{ng} / \mathrm{ml})$, insufficient $(11-20 \mathrm{ng} / \mathrm{ml})$, and deficient $(\leq 10 \mathrm{ng} / \mathrm{ml})$. We also tried to assess the correlation between vitamin $\mathrm{D}$ status and the severity and frequency of headache attacks. Results: In the migraine group, five patients ( 3 girls and 2 boys) had optimal vitamin D, 20 (10 girls and 10 boys) had insufficiency and five ( 4 girls and 1 boy) were deficient. In the control group, six patients ( 4 girls and 2 boys) had optimal vitamin D, 19 (8 girls and 11 boys) had insufficiency, and five (4 girls and 1 boy) were deficient. Vitamin D status had no significant difference between groups $(p=0.943)$. We could not find any correlation between vitamin D status and severity (Pearson correlation, $p=0.513$ ) and frequency (Pearson correlation, $p=0.422$ ) of migraine attacks.

Conclusions: Vitamin D insufficiency and deficiency are equally common in children with migraine and healthy controls. We need well designed case control studies with large sample sizes to properly assess the probable pathophysiologic role of vitamin D in children with migraine.

\section{KEY WORDS:}

children, migraine, vitamin D status, case control.

\section{INTRODUCTION}

Migraine is very common in children and adolescents, affecting about $4-11 \%$ of children in elementary schools and $8-23 \%$ of adolescents [1]. According to the criteria of the international classification of headache disorders, $3^{\text {rd }}$ edition (ICHD-III) [2], migraine headaches in children are usually characterized by attacks of pulsating and uni- lateral or bilateral headaches with photo-phonophobia, and nausea or vomiting. These headaches last 2-72 hours and the behaviour of children usually changes during the headaches. Migraine headaches have a huge negative impact on the quality of life in children and their families [1]. Although bio-behavioural measures such as adequate sleep and appropriate and on time meals may help managing headaches, many children with frequent

\section{ADDRESS FOR CORRESPONDENCE:}

Reza Azizimalamiri, Department of Pediatrics, division of pediatric neurology, Golestan Medical, Educational, and Research Center, Ahvaz Jundishapur University of Medical Sciences, Ahvaz, Iran, Tel./Fax: +98 613 3743063, ORCID: https://orcid.org/0000-0001-5423-6375, e-mail: azizi-r@ajums.ac.ir, azizi.ramin@gmail.com 
(more than 2 attacks per week) or severe migraine attacks require medications [3].

Medications approved by FDA to administer in children with migraine are limited. A number of different classes of medications have been used including antiseizure medications (valproate, levetiracetam), calcium channel blockers (flunarizine, cinnarizine, cyproheptadine), beta-blockers (propranolol), and tricyclic antidepressants (amitriptyline) [3].

In addition to the mentioned medications, a number of dietary supplements such as minerals and vitamins have been suggested to prevent recurrent migraine attacks. Liampas et al. have been conducted 4 important systematic reviews and meta-analyses and provided the evidence for the use of complementary approaches [4-7]. In these studies, the effects of supplemental pyridoxine, folate, cobalamine, and the serum homocysteine in patients with migraine have been evaluated and possible therapeutic roles have been suggested $[6,7]$. They also assessed the therapeutic use of exogenous melatonin in migraine [5]. Among the different vitamins, one of the most noted is vitamin $\mathrm{D}[4,8]$.

Vitamin $\mathrm{D}$ has a wide range of effects on different systems of the human body including the nervous system [8]. Some observational studies and a few clinical trials tried to show the role of this vitamin in migraine headaches [8-24], however, the results are controversial and prescribing vitamin $\mathrm{D}$ in every patient with migraine is not indicated [8]. Vitamin D may influence migraine headaches in a number of ways. Inflammation plays a key role in migraine; hence, the anti-inflammatory role of vitamin D may play an important role in preventing migraine headaches [25]. In addition, vitamin D supplementation can reduce inflammatory factors such as $\mathrm{C}$ reactive protein as a strong inflammatory mediator [26]. Other possible pathophysiologic roles of vitamin $\mathrm{D}$ in migraine headaches are related to its effects on the possible sensitization of the second and third neurons. These neurons are connected with the stimulation of sensory receptors of the periosteal covering and central sensitization [17,27]. Vitamin D deficiency could also lead to magnesium deficiency. Magnesium has a central role in inhibiting excessive excitation and studies have shown a strong association between migraine and magnesium deficiency [28]. Nitric oxide (NO) is known as a key mediator in migraine and vitamin D reduces the production of NO [29]. The release of dopamine and serotonin have been shown to be influenced by vitamin $\mathrm{D}$ and these factors are known to be connected with the pathogenesis of migraine [30]. Low levels of melatonin have been shown in patients with migraine and vitamin $\mathrm{D}$ deficiency could lead to low melatonin levels [31]. All of these suggested mechanisms indicate the importance of vitamin $\mathrm{D}$ in the pathophysiology of migraine.

To our knowledge, no study has been conducted specifically in Iran to show the vitamin D status in children with migraine; therefore, we conducted a study to assess the status of vitamin $\mathrm{D}$ in children with migraine and comparing them with a healthy age- and sex-matched healthy controls. We also tried the assess the correlation between vitamin D serum levels and the severity and frequency of headaches.

\section{MATERIAL AND METHODS}

\section{STUDY DESIGN, LOCATION, AND PARTICIPANTS}

We conducted a prospective case-control study. The study was performed at Golestan Hospital, a University-affiliated referral hospital in Ahvaz, Southwest of Iran. The study began on June 1, 2018, and continued until November 30, 2019. Eligible children were those who aged 6-14 years that according to the international classification of headache disorders, $3^{\text {rd }}$ edition (ICHD-III) criteria had migraine (with and without aura) [2]. A child neurologist confirmed the migraine diagnosis and all the children in the migraine group had headaches at least more than 6 months. To rule out other causes of headaches, a detailed history was taken and thorough systemic and neurologic examinations were conducted. Brain imaging (MRI) was conducted in doubtful cases. After obtaining written informed consent, participants were consecutively enrolled to the migraine or control group. Participants in the control group composed of healthy children who were referred for growth and development monitoring. All the children in the control group were healthy without any history of migraine headaches or other primary headache disorders. To increase the betweengroups comparability, we recruited them from the same schools and living areas as the migraine group.

\section{INCLUSION AND EXCLUSION CRITERIA}

Included children (6-14 years) were those with migraine (with and without aura) and a group of sex- and age-matched healthy children as a control group. Those who did not give consent, either themselves or their parents, were excluded. We also excluded all those who had major comorbidities such as diabetes mellitus, renal failure, epilepsy, congenital heart disease, malignancy, rheumatologic disorders, gastrointestinal diseases, and those with clinical manifestations of rickets or osteomalacia and those who were taking supplemental vitamin D or any medication that could change the metabolism of vitamin $\mathrm{D}$ and calcium. All of these conditions may have a direct or indirect effect on vitamin D status.

\section{SAMPLING}

We referred all the participants to the central laboratory of the centre for blood sampling from 8:00 to 10:00 A.M. every day except Friday. In each participant, a 5-ml blood sample was collected under standard aseptic con- 
TABLE 1. Baseline characteristics; migraine group $(n=30)$

\begin{tabular}{|l|c|c|}
\hline Characteristics & Boys & Girls \\
\hline Gender & $13(43.3 \%)$ & $17(56.4 \%)$ \\
\hline Age, mean \pm SD & $8.8 \pm 2.7$ & $8.7 \pm 2.8$ \\
\hline Age, median (range) & $9(6-14)$ & $9(6-14)$ \\
\hline Body mass index (mean \pm SD) & $19.2 \pm 3.8$ & $19.4 \pm 6.1$ \\
\hline Positive family history of migraine & 9 from 13 & 17 \\
\hline Nausea and vomiting & 9 from 13 & 5 from 17 \\
\hline Abdominal pain & 4 from 13 & 5 from 17 \\
\hline Phonophobia & 2 from 13 & 7 from 17 \\
\hline Photophobia & 8 from 13 & 10 from 17 \\
\hline $\begin{array}{l}\text { Headache frequency (per month), } \\
\text { median (range) }\end{array}$ & $11(4-30)$ & $12(4-30)$ \\
\hline $\begin{array}{l}\text { Headache duration (in hours), } \\
\text { median (range) }\end{array}$ & $2(1-72)$ & $4.5(1-24)$ \\
\hline Headache severity, median (range) & $8(3-10)$ & $8(3-10)$ \\
\hline Presence of aura (mostly visual) & $4 / 13$ & $6 / 17$ \\
\hline
\end{tabular}

TABLE 2. Baseline characteristics; control group $(n=30)$

\begin{tabular}{|l|c|c|}
\hline Characteristics & Boys & Girls \\
\hline Gender & $14(46.6 \%)$ & $16(54.4 \%)$ \\
\hline Age, mean \pm SD & $8.8 \pm 2.9$ & $8.8 \pm 2.7$ \\
\hline Age, median (range) & $9(6-14)$ & $9(6-14)$ \\
\hline Body mass index (mean \pm SD) & $16.5 \pm 2.4$ & $17.1 \pm 2.4$ \\
\hline
\end{tabular}

dition. The samples immediately were centrifuged then serums of the samples were frozen and kept at $-70^{\circ} \mathrm{C}$. Plasma levels of 25(OH)D were measured using a chemiluminescent immunoassay kit [" $25(\mathrm{OH}) \mathrm{D}$ total assay, Diasorin Liaison"] by LIAISON analyser. The best way of defining vitamin $\mathrm{D}$ status in a person is measuring the prohormone $25(\mathrm{OH}) \mathrm{D}$, which is an indicator of supply rather than function [32]. Vitamin D status was classified as Optimal (> $20 \mathrm{ng} / \mathrm{ml})$, insufficient $(11-20 \mathrm{ng} / \mathrm{ml})$, and deficient $(\leq 10 \mathrm{ng} / \mathrm{ml})$ [32]. In children with migraine the frequency, duration, and severity of headaches were registered. We asked all the children with migraine and their parents to record the frequency (per month), severity (using the scale), and duration (in hours) of headaches in previously prepared charts during the next month after enrolment. To assess the severity of headaches, we used the Wong-Baker FACES Pain Rating Scale [33, 34]. Wong-Baker FACES Pain Rating Scale ranges from 0 to 10 . On this scale, 0 equals no pain, $1-3$ equals mild pain, 4-6 equals moderate pain, and 7-10 equals severe pain. According to the literature, the frequency of headaches could be categorized; fewer than seven headache days per month (low-frequency episodic migraine), 7-14 days per month (high-frequency episodic migraine), and 15 or more days per month (chronic migraine)[35].

\section{STATISTICAL ANALYSIS}

According to the previous studies $[12,15,23]$ and based on the type I error $\alpha=0.05$ and power of $80 \%$ we needed at least 30 children in each group to detect differences between the serum vitamin D levels of the two groups. The Kolmogorov-Smirnov test was used to define the normality of the data. We used independent sample $t$-test and analysis of variance (ANOVA) to analysed continuous data. Categorical variables were analysed using the $\chi^{2}$ test. We used Pearson correlation test to assess the correlation of vitamin $\mathrm{D}$ serum levels and the severity and frequency of headaches. The $p$-values $<0.05$ was considered significant.

\section{ETHICS}

The study design and its protocol have been assessed and approved by the ethics committee of the centre and Ahvaz Jundishapur University of Medical Sciences (D/3265) and we performed the study in accordance with the ethical standards as laid down in the 1964 Declaration of Helsinki and its later amendments. All the parents and their children read and signed written informed consent before enrolment. Verbal consent was obtained from children who could not sign our forms.

\section{RESULTS}

\section{BASELINE CHARACTERISTICS}

In this study, 50 children with migraine and 45 healthy control were eligible to enrol. However, in the migraine group, 10 children did not give consent, 8 children had no cooperation to record the requested data, and 2 were lost to follow up. In the control group, 15 children did not give consent. Sequentially, we enrolled 60 children with confirmed eligibility (30 children in each group) into the study and the data from all of them were analysed. The baseline characteristics of the participants are summarized in Table 1 (migraine group) and Table 2 (control group). The age range of the participants was between 6-14 years and the median age in both groups was 9 years. Migraine characteristics such as frequency, duration, and severity of headaches are shown in Table 1. Migraine accompaniments also are shown in Table 1. In terms of age, gender, and body mass index, no statistically significant differences were found between groups (Table 3). None of the children from both groups showed the signs and symptoms of vitamin deficiency or insufficiency. Table 3 compares groups regarding age, gender, and weight. 


\section{VITAMIN D STATUS IN THE PARTICIPANTS, AND ITS CORRELATION WITH HEADACHE SEVERITY AND FREQUENCY}

The mean \pm SD of vitamin D serum levels $(\mathrm{ng} / \mathrm{ml})$ was $(15.9 \pm 5.4)$ in migraine group and $(17.4 \pm 11.3)$ in healthy controls. Independent sample $t$-test showed no significant difference between groups (two tailed independent sample $t$-test, $p$-value $=0.509$ ). As shown in Table 4 , in the migraine group five patients ( 3 girls and 2 boys) had optimal levels of vitamin D, 20 (10 girls and 10 boys) had insufficiency, and five ( 4 girls and 1 boy) were deficient. In the control group, six patients ( 4 girls and 2 boys) had optimal levels of vitamin D, 19 (8 girls and 11 boys) had insufficiency, and five ( 4 girls and 1 boy) were deficient. Vitamin D status had no significant difference between both groups $\left(\chi^{2}\right.$, $p=0.943$ ). We could not find any correlation between vitamin D status and severity (Pearson correlation, $p=0.513$ ) and frequency (Pearson correlation, $p=0.422$ ) of migraine attacks. Table 5 shows the vitamin D serum levels $(\mathrm{ng} / \mathrm{ml})$ and compares it between groups regarding gender, age groups, the presence of aura, and severity and frequency of headaches. As shown in Table 5, no statistically significant difference in serum vitamin D levels was found between groups regarding age groups and gender. In addition, the serum vitamin D levels was not significantly different in migraine groups regarding presence or absence of aura, and the frequency and severity of headaches.

\section{DISCUSSION}

The results of this study indicate that vitamin D insufficiency and deficiency in children with migraine are as common as in healthy children. Our results did not indicate any correlation between vitamin $\mathrm{D}$ serum values and the severity and frequency of headaches in children. These results may be inclined by small sample size of the study. We know that to define delicate differences we need large sample sizes.

Previous studies of vitamin D status in patients with migraine had controversial results. A number of case-control studies proposed an inverse correlation between the vitamin D serum levels and the severity of headaches [12, 23]. However, these studies could not indicate plausible pathophysiologic evidence for their claims that vitamin D insufficiency or deficiency essentially worsens migraine headaches. Like observational studies, clinical trials of vitamin D supplementation in patients with migraine had controversial results $[10,11,13,14,16]$. A few clinical trials showed the positive effect of vitamin D supplementation on the frequency and severity of headaches while other experimental studies showed no effect of administering vitamin D. Among these trials, the study conducted by Knutsen et al. did not show any significant difference between vitamin supplementation and the placebo effect on the occurrence, anatomical localization, and degree of pain or headache [16]. In contrast, studies conduct-
TABLE 3. Comparison between gender, age, and weight of the participants

\begin{tabular}{|l|c|c|c|}
\hline Characteristics & $\begin{array}{c}\text { Migraine } \\
\text { group }\end{array}$ & Controls & $p$-value \\
\hline Boys & 13 & 14 & $1^{*}$ \\
\hline Girls & 17 & 16 & - \\
\hline Age (mean \pm SD) & $8.8 \pm 2.7$ & $8.8 \pm 2.7$ & $1^{* *}$ \\
\hline Weight (mean \pm SD) & $33.4 \pm 14.1$ & $34.2 \pm 12.6$ & $0.818^{* *}$ \\
\hline
\end{tabular}

${ }^{*} \chi^{2}$ test, ${ }^{* *}$ Student's $t$-test

TABLE 4. Vitamin D status in both groups

\begin{tabular}{|l|c|c|c|}
\hline Vitamin D status & $\begin{array}{c}\text { Migraine } \\
\text { group } \\
(n=30)\end{array}$ & $\begin{array}{c}\text { Control } \\
\text { group } \\
(n=30)\end{array}$ & $p$-value* \\
\hline Optimal $(>20 \mathrm{ng} / \mathrm{ml})$ & 5 & 6 & 0.943 \\
\hline Insufficient $(11-20 \mathrm{ng} / \mathrm{ml})$ & 20 & 19 & - \\
\hline Deficient $(\leq 10 \mathrm{ng} / \mathrm{ml})$ & 5 & 5 & - \\
\hline${ }^{*} \chi^{2}$ test
\end{tabular}

TABLE 5. Comparison of vitamin D serum levels $(\mathrm{ng} / \mathrm{ml})$ (mean \pm SD) between groups regarding gender, age groups, presence of aura, severity and frequency of headaches

\begin{tabular}{|l|c|c|c|}
\hline Characteristics & $\begin{array}{c}\text { Migraine } \\
\text { group }\end{array}$ & Controls & $p$-value \\
\hline Boys & $14 \pm 5.4$ & $20.2 \pm 12.6$ & $0.129^{*}$ \\
\hline Girls & $18.9 \pm 4$ & $15.6 \pm 10.2$ & $0.237^{*}$ \\
\hline
\end{tabular}

Age groups (in years)

\begin{tabular}{|l|c|c|c|}
\hline $6-9$ & $16.1 \pm 4.3$ & $16.5 \pm 11.3$ & $0.906^{*}$ \\
\hline $10-12$ & $16.1 \pm 7.1$ & $21.2 \pm 12.6$ & $0.399^{*}$ \\
\hline$>12$ & $18.1 \pm 6.1$ & $18.2 \pm 7.3$ & $0.988^{*}$ \\
\hline
\end{tabular}

Presence of aura

\begin{tabular}{|l|c|c|c|}
\hline Yes & $18.2 \pm 6.2$ & - & $0.988^{*}$ \\
\cline { 1 - 3 } No & $18.4 \pm 7.1$ & - & \\
\cline { 1 - 3 } &
\end{tabular}

Severity of headaches (see text)

\begin{tabular}{|l|c|c|c|}
\hline $\begin{array}{l}\text { Mild (1-3) } \\
\text { (3 patients) }\end{array}$ & $15.5 \pm 7.3$ & - & $0.950^{* *}$ \\
\hline $\begin{array}{l}\text { Moderate (4-6) } \\
\text { (18 patients) }\end{array}$ & $15.7 \pm 5.9$ & - & \\
\hline $\begin{array}{l}\text { Severe (7-10) } \\
\text { (9 patients) }\end{array}$ & $17.6 \pm 3.5$ & - & \\
\hline
\end{tabular}

\begin{tabular}{|l|c|c|c|}
\hline \multicolumn{3}{|l|}{ Frequency of headaches (days per month) } \\
\hline$<>7$ (14 patients) & $15.2 \pm 5.3$ & - & $0.950^{* * *}$ \\
\hline $7-14$ (10 patients) & $17.6 \pm 3.5$ & - & \\
\hline$\geq 15$ (6 patients) & $15.7 \pm 5.9$ & - & \\
\hline
\end{tabular}

*Independent sample t-test, **One way analysis of variance 
ed by Ghorbani et al., Gazerani et al., and Buettner et al. showed the efficacy of supplemental vitamin D in patients with migraine $[10,13,14]$.

Among previous studies, Hanc1 et al. conducted a case-control study in children with primary headaches [15]. The age range in their study (5-17 years) was almost similar to the age range of ours. Interestingly, their results are comparable to our results. Like us, they did not find any difference in vitamin $\mathrm{D}$ status between children with migraine and healthy controls. In contrast to our results, the results of a case-control study in children that was conducted by Donmez et al. indicated that vitamin D levels in children with migraine were significantly lower than the healthy control group [12]. Another case-control study conducted by Tozzi et al. indicated a high incidence of vitamin D deficiency in the paediatric population with headache (56\%), particularly in migraineurs without aura [23]. All the remaining observational studies were conducted in young adults. Although these studies in the adults had controversial results, a recent meta-analysis conducted by Liampas et al. showed interesting results. This study pooled the data from the nine eligible studies. Serum levels of $25(\mathrm{OH}) \mathrm{D}$ were determined significantly lower in migraine patients $(n=952)$ in comparison with healthy controls $(n=8013)$ (eight studies, PQ $<0.1, I^{2}=94 \%$, RE model MD $=-4.11,95 \% \mathrm{CI}:-6.48$ to -1.74$)$. The authors indicated that investigation of the effect of vitamin D supplementation in patients suffering from migraine is warranted [4].

The pathophysiology of migraine is complex and until now the main involved mechanisms in this condition have not been fully elucidated. A number of studies proposed possible role of calcium in the pathophysiology of migraine. These studies have been suggested the possible role of hypercalcemia in migraine headaches and proposed that genetically elevated serum calcium over lifetime appears to increase risk for migraine [36]. Moreover, investigators have had attempted to explain a number of pathophysiologic mechanisms of vitamin D and its deficiency in patients with migraine [8]. Among these mechanisms, anti-inflammatory effects of calcitriol, controlling $\mathrm{T}$ helper cells, acting as a neuroactive steroid, inhibiting nitric oxide, and suppressing prostaglandin E2 are the most proposed mechanisms [8]. In addition, clinical trials of supplemental vitamin $\mathrm{D}$ in patients with migraine headaches have shown controversial results, and supplementing with vitamin D is not indicated in all patients with migraine. By the way, a highly selected group of patients with migraine and vitamin $\mathrm{D}$ deficiency might benefit from supplemental vitamin $\mathrm{D}$ [8].

Jazayeri et al. conducted a meta-analysis and studied the prevalence of vitamin deficiency and insufficiency in Iranian children [37]. They pooled the data from the 11 studies from all over the country. The prevalence of vitamin D deficiency in Iranian boys and girls were 35\% (95\% CI: 34-37) and 61\% (95\% CI: 60-63), respectively. The prevalence of vitamin D insufficiency in Iranian chil- dren and adolescents was 31\% (95\% CI: 30-31). Although the results of our study are somewhat different from the results of this meta-analysis but when we compared our results with the individual studies from Iran, we found that our results are close to the results of the study conducted by Mellati et al. [38]. Iran is a vast country and the exposure of people to sunlight is different over the country. Our province, Khuzestan, is sunny and people are exposed to sunlight more than other areas of the country, however, dietary insufficiency of vitamin D supplements could result in insufficient production of vitamin D after exposure to sunlight but could prevent from severe deficiency. It seems that the results of our study could represent the vitamin D status from this part of our country.

The results of our study should be interpreted in the face of certain limits. The sample size of our study was small. Our patients were from the Southwest of Iran; therefore, our results should not be generalized to other provinces of Iran. In addition, studies showed that the prevalence of vitamin D insufficiency and deficiency in Iran is high [37]; therefore, any study of assessing vitamin D status in any population from Iran could be affected by this high prevalence.

\section{CONCLUSIONS}

Vitamin D insufficiency and deficiency are common in children and it seems that its prevalence is similar in children with migraine and healthy controls. To determine the role of vitamin D in the pathophysiology of migraine or its management, we need well-designed clinical trials of supplementing vitamin $\mathrm{D}$ in children with migraine who suffer from vitamin D deficiency or insufficiency.

\section{ACKNOWLEDGEMENTS}

This paper is from the thesis of Sahar Majidinezjad, M.D. The Research Deputy of Ahvaz Jundishapur University of Medical Sciences provided financial and logistic support for this study (grant number: U-96159), but had no role in study design; data collection, analysis, or interpretation; writing the report; or in the decision to submit the article for publication. The authors are grateful to all parents and patients for their participation in this study. We are extremely indebted to the authorities of the Research Deputy of Ahvaz Jundishapur University of Medical Sciences for their financial and logistic support.

\section{DISCLOSURE}

The authors declare no conflict of interest.

\section{REFERENCES}

1. Lewis DW. Pediatric migraine. Neurol Clin 2009; 27: 481-501.

2. Headache Classification Committee of the International Headache Society (IHS). The International Classification of Headache Disorders, $3^{\text {rd }}$ ed. Cephalalgia 2018; 38: 1-211. 
3. El-Chammas K, Keyes J, Thompson N, et al. Pharmacologic treatment of pediatric headaches: a meta-analysis. JAMA Pediatr 2013; 167: 250-258.

4. Liampas I, Siokas V, Brotis A, Dardiotis E. Vitamin D serum levels in patients with migraine: a meta-analysis. Rev Neurol (Paris) 2020; 176: 560-570.

5. Liampas I, Siokas V, Brotis A, et al. Endogenous melatonin levels and therapeutic use of exogenous melatonin in migraine: systematic review and meta-analysis. Headache 2020; 60: 1273-1299.

6. Liampas I, Siokas V, Mentis AA, et al. Serum homocysteine, pyridoxine, folate, and vitamin B12 levels in migraine: systematic review and meta-analysis. Headache 2020; 60: 1508-1534.

7. Liampas IN, Siokas V, Aloizou AM, et al. Pyridoxine, folate and cobalamin for migraine: a systematic review. Acta Neurol Scand 2020; 142: 108-120.

8. Nowaczewska M, Wicinski M, Osinski S, Kazmierczak H. The role of vitamin $\mathrm{d}$ in primary headache-from potential mechanism to treatment. Nutrients 2020; 12: 243.

9. Buettner C, Burstein R. Association of statin use and risk for severe headache or migraine by serum vitamin D status: a cross-sectional population-based study. Cephalalgia 2015; 35: 757-766.

10. Buettner C, Nir RR, Bertisch SM, et al. Simvastatin and vitamin D for migraine prevention: a randomized, controlled trial. Ann Neurol 2015; 78: 970-981.

11. Cayir A, Turan MI, Tan H. Effect of vitamin D therapy in addition to amitriptyline on migraine attacks in pediatric patients. Braz J Med Biol Res 2014; 47: 349-354.

12. Donmez A, Orun E, Sonmez FM. Vitamin D status in children with headache: a case-control study. Clin Nutr ESPEN 2018; 23: 222-227.

13. Gazerani P, Fuglsang R, Pedersen JG, et al. A randomized, doubleblinded, placebo-controlled, parallel trial of vitamin D3 supplementation in adult patients with migraine. Curr Med Res Opin 2019; 35: 715-723.

14. Ghorbani Z, Rafiee P, Fotouhi A, et al. The effects of vitamin D supplementation on interictal serum levels of calcitonin gene-related peptide (CGRP) in episodic migraine patients: post hoc analysis of a randomized double-blind placebo-controlled trial. J Headache Pain 2020; 21: 22.

15. Hanci F, Kabakus N, Turay S, et al. The role of obesity and vitamin $D$ deficiency in primary headaches in childhood. Acta Neurol Belg 2020; 120: 1123-1131.

16. Knutsen KV, Madar AA, Brekke M, et al. Effect of vitamin D on musculoskeletal pain and headache: a randomized, double-blind, placebo-controlled trial among adult ethnic minorities in Norway. Pain 2014; 155: 2591-2598.

17. Mottaghi T, Khorvash F, Askari G, et al. The relationship between serum levels of vitamin D and migraine. J Res Med Sci 2013; 18: S66-70.

18. Prakash S, Kumar M, Belani P, et al. Interrelationships between chronic tension-type headache, musculoskeletal pain, and vitamin D deficiency: is osteomalacia responsible for both headache and musculoskeletal pain? Ann Indian Acad Neurol 2013; 16: 650-658.

19. Prakash S, Rathore C, Makwana P, et al. Vitamin D deficiency in patients with chronic tension-type headache: a case-control study. Headache 2017; 57: 1096-1108.

20. Rapisarda L, Mazza MR, Tosto F, et al. Relationship between severity of migraine and vitamin $\mathrm{D}$ deficiency: a case-control study. Neurol Sci 2018; 39: 167-168.

21. Song TJ, Chu MK, Sohn JH, et al. Effect of vitamin D deficiency on the frequency of headaches in migraine. J Clin Neurol 2018; 14: 366-373.

22. Togha M, Razeghi Jahromi S, Ghorbani Z, et al. Serum vitamin D status in a group of migraine patients compared with healthy controls: a case-control study. Headache 2018; 58: 1530-1540.
23. Tozzi E, Boncristiano A, Antenucci A, et al. P013. 25(OH)D level and headache in children sample. J Headache Pain 2015; 16: A84.

24. Zandifar A, Masjedi SS, Banihashemi M, et al. Vitamin D status in migraine patients: a case- control study. Biomed Res Int 2014; 2014: 514782.

25. Burstein R, Noseda R, Borsook D. Migraine: multiple processes, complex pathophysiology. J Neurosci 2015; 35: 6619-6629.

26. Mottaghi T, Askari G, Khorvash F, Maracy MR. Effect of Vitamin D supplementation on symptoms and C-reactive protein in migraine patients. J Res Med Sci 2015; 20: 477-482.

27. Kenis-Coskun O, Giray E, Gunduz OH, Akyuz G. The effect of vitamin D replacement on spinal inhibitory pathways in women with chronic widespread pain. J Steroid Biochem Mol Biol 2020; 196: 105488.

28. Kirkland AE, Sarlo GL, Holton KF. The role of magnesium in neurological disorders. Nutrients 2018; 10: 730.

29. Messlinger K, Lennerz JK, Eberhardt M, Fischer MJ. CGRP and NO in the trigeminal system: mechanisms and role in headache generation. Headache 2012; 52: 1411-1427.

30. Goadsby PJ, Holland PR. An update: pathophysiology of migraine. Neurol Clin 2019; 37: 651-671.

31. Sohn JH, Chu MK, Park KY, et al. Vitamin D deficiency in patients with cluster headache: a preliminary study. J Headache Pain 2018; 19: 54 .

32. Thacher TD, Clarke BL. Vitamin D insufficiency. Mayo Clin Proc 2011; 86: 50-60.

33. Wong DL, Baker CM. Pain in children: comparison of assessment scales. Pediatr Nurs 1988; 14: 9-17.

34. Mittal S, Sharma A, Sharma A, et al. Banded versus single-sided bonded space maintainers: a comparative study. Indian Journal of Dental Sciences 2018; 10: 29-36.

35. Buse DC, Manack A, Serrano D, et al. Sociodemographic and comorbidity profiles of chronic migraine and episodic migraine sufferers. J Neurol Neurosurg Psychiatry 2010; 81: 428-432.

36. Yin P, Anttila V, Siewert KM, et al. Serum calcium and risk of migraine: a Mendelian randomization study. Hum Mol Genet 2017; 26: 820-828.

37. Jazayeri M, Moradi Y, Rasti A, et al. Prevalence of vitamin D deficiency in healthy Iranian children: a systematic review and meta-analysis. Med J Islam Repub Iran 2018; 32: 83.

38. Mellati AA, Sharifi F, Faghihzade S, et al. Vitamin D status and its associations with components of metabolic syndrome in healthy children. J Pediatr Endocrinol Metab 2015; 28: 641-648. 